\title{
Suprimento arterial para a bolsa cloacal em aves (Gallus gallus Lin- naeus, 1758) da linhagem Lhoman White LSL
}

\author{
Eduardo Maurício Mendes de Lima ${ }^{1 *}$ \\ Frederico Ozanam Carneiro e Silva ${ }^{2}$ \\ Renato Souto Severino ${ }^{2}$ \\ Sérgio Salazar Drummond ${ }^{2}$ \\ Cleide Lúcia Araújo² \\ Pedro Primo Bombonato ${ }^{3}$ \\ Marcelo Ismar Silva Santana ${ }^{4}$ \\ ${ }^{1}$ Faculdade de Agronomia e Medicina Veterinária da Universidade de Brasília \\ Instituto Central de Ciências Ala Sul, Campus Universitário Darcy Ribeiro \\ Caixa Postal 4508, CEP 70910-970, Brasília - DF, Brasil \\ ${ }^{2}$ Faculdade de Medicina Veterinária da Universidade Federal de Uberlândia \\ ${ }^{3}$ Faculdade de Medicina Veterinária e Zootecnia da Universidade de São Paulo \\ ${ }^{4}$ Pontíficia Universidade Católica de Minas Gerais, Poços de Caldas \\ *Autor para correspondência \\ limaemm@unb.br
}

\section{Resumo}

Foram estudados a origem, o número e a ordenação dos ramos arteriais que suprem a bolsa cloacal em 30 exemplares de Gallus gallus, da linhagem Lohman White LSL, fêmeas, com 8 a 10 semanas de idade. Estas aves tiveram seu sistema arterial preenchido com solução aquosa a 50\% de Neoprene Látex " 450 ”, corada com pigmento específico, em seguida foram fixadas em solução aquosa de formol a $10 \%$ e submetidas à dissecação. Verificou-se que a bolsa cloacal é suprida por ramos diretos das artérias bursocloacal direita, em 29 casos (96,67\%), bursocloacal esquerda, em 28 casos (93,33\%), cloacal direita, em 13 casos (43,33\%) e cloacal esquerda, em 15 casos (50\%). O número de ramos cedidos pelas artérias, independente da sua origem, variou de 2 a 6 ramos, sendo dois ramos em 5 casos (16,67\%), três ramos em 14 casos (46,67\%), quatro ramos em 3 casos (10\%), cinco ramos em 6 casos $(20 \%)$ e seis ramos em 1 caso $(3,33 \%)$. Foi possível observar que a distribuição dos vasos arteriais que suprem a bolsa cloacal deu-se de maneira própria para cada um dos exemplares investigados.

Unitermos: Gallus gallus, bolsa cloacal, artérias, linhagem Lhoman White LSL

\section{Abstract}

Arterial supply to the cloacal bursa of the Lhoman White LSL lineage fowl (Gallus gallus Linnaeus, 1758). A study has been carried out on the origin, number and disposition of the arterial branches supplying the cloacal bursas of 30 female fowl (Gallus gallus) of the Lhoman White LSL lineage, aged between eight and ten 
weeks. The right isquiadic artery was cannulated and injected with a 50\% colored solution of Neoprene latex " 450 ". Next, the samples were fixed with a $10 \%$ formalin solution through deep intramuscular, subcutaneous and intracavitary injections for a period of at least 48 hours. Dissections confirmed that the cloacal bursa was supplied by direct branches from the right bursocloacal arteries in 29 cases (96.67\%), the left bursocloacal arteries in 28 cases $(93.33 \%)$, the right cloacal arteries in 13 cases $(43.33 \%)$, and the left cloacal arteries in 15 cases $(50 \%)$. The number of branches from the arteries, independent of their origin, varied from two to six. There were two branches in 5 cases $(16.67 \%)$, three branches in 14 cases $(46.67 \%)$, four branches in 3 cases $(10 \%)$, five branches in 6 cases $(20 \%)$, and six branches in 1 case $(3.33 \%)$. The distribution of the vessels was unique in each sample.

Key words: Gallus gallus, cloacal bursa, arteries, Lhoman White LSL lineage

\section{Introdução}

A contribuição da bolsa cloacal para o desenvolvimento de uma resposta imune nas aves deve ser enfatizada, uma vez que, juntamente com o timo, responde pela maturação e transferência de linfócitos para outros sistemas.

No tocante à avicultura, observa-se que esta apresenta grandes avanços nos conhecimentos relativos à biosseguridade, principalmente no que diz respeito aos esquemas de vacinações e prevenção de doenças, onde um melhor conhecimento a respeito das respostas imunológicas tem sido freqüentemente abordado (Payne, 1971).

Destaca-se que os mecanismos que regulam o desenvolvimento da bolsa cloacal, assim como a linfocitopoiese, não estão definitivamente esclarecidos. Assim, deve ser ressaltada a necessidade do empreendimento de conhecimentos específicos a respeito deste órgão, devido à sua considerável importância no complexo imunitário. Garantindo a necessidade básica do conhecimento de sua morfologia, abordada é claro de maneira sistemática, em especial no que diz respeito aos componentes vasculares que promovem seu suprimento sangüíneo.

Assim, os informes de Schwarze e Schröder (1970), Zamojska (1975), Nickel et al. (1981) e Baumel (1993) promoveram uma abordagem específica a respeito dos padrões vasculares relativos ao suprimento sangüíneo para a bolsa cloacal em aves do gênero Gallus. De outra forma foi possível ainda encontrar os informes de Baumel (1988) relativos ao suprimento arterial da bolsa cloacal em pombos, os de Scala et al. (1989) para patos, e os de Onyeanusi et al. (1993) referindo-se à galinha d'Angola.
Em outro instante foram compilados os estudos específicos em Gallus gallus, como os de Silva et al. (1997; 2001 e 2003), Santana et al. (1999; 2000 e 2003) e Lima et al. (2002), que contribuíram de maneira mais detalhada sobre os dados relativos ao número, a origem e distribuição dos ramos arteriais destinados à bolsa cloacal em diferentes linhagens.

Buscando melhor esclarecer os diversos aspectos ainda controvertidos, no tocante aos ramos arteriais responsáveis pelo suprimento sangüíneo da bolsa cloacal, e ainda visando contribuir com a anatomia comparativa, o presente estudo teve como objetivo retratar de maneira sistemática os aspectos concernentes ao número, à origem e à distribuição das artérias que suprem a bolsa cloacal em aves (Gallus gallus Linnaeus, 1758) da linhagem Lhoman White LSL.

\section{Material e Métodos}

Para a realização deste trabalho, foram utilizados 30 exemplares de aves (Gallus gallus Linnaeus, 1758) da linhagem Lohman White LSL, fêmeas, com 8 a 10 semanas de idade.

Para eutanásia das aves, empregou-se o protocolo padrão sugerido por Rosskpof e Woerpel (1966), ou seja, a utilização de alta dose de gás anestésico (Halotane, Laboratório Cristália), 10\%, durante três minutos.

Com a finalidade de marcar o sistema arterial dos exemplares, foi promovida canulação da artéria isquiática direita de cada um destes e, em seguida, preencheuse este sistema com solução aquosa a $50 \%$ de Neoprene Látex “450” (Du Pont do Brasil Indústrias Químicas), corada com pigmento específico (Globo S/A Tintas e Pigmentos). 
A seguir, as aves foram fixadas com solução aquosa a $10 \%$ de formol (Labsynth - Produtos para Laboratórios Ltda), mediante aplicação intramuscular profunda, subcutânea e intracavitária, sendo posteriormente mantidas submersas na mesma solução em recipientes adequados, por período mínimo de 48 horas.

Para dissecação dos vasos responsáveis pelo suprimento arterial da bolsa cloacal empregou-se campo visual de uma lupa monocular do tipo Wild (10x). No entanto, para visualização do órgão e dos vasos responsáveis pelo seu suprimento sangüíneo, foram efetuadas duas incisões paramedianas na base do pigóstilo, divulsionando-se e retirando os tecidos adiposo e conjuntivo dispostos entre este e a abertura cloacal para, então, alcançar as artérias responsáveis pelo suprimento do órgão.

Foi realizada fotografia (Figura 1) ilustrando a origem, o número e a ordenação das artérias responsáveis pelo suprimento sangüíneo da bolsa cloacal.

\section{Resultados}

A bolsa cloacal em aves da linhagem Lhoman White LSL mostrou-se suprida, independentemente do número e do seu território, pelas artérias bursocloacais e artérias cloacais de ambos os antímeros.

No referente às origens das artérias envolvidas, em todos os exemplares dissecados, observou-se serem as artérias pudendas internas, direita e esquerda, originárias das artérias ilíacas internas correspondentes, que apresentaram como colaterais as artérias bursocloacais direita e esquerda. A bolsa cloacal foi suprida por ramos diretos das artérias bursocloacal direita em 29 casos $(96,67 \%)$, sendo que um ramo esteve presente em 23 casos (76,67\%), dois ramos em quatro casos $(13,33 \%)$ e três ramos em dois casos $(6,67 \%)$. A artéria bursocloacal esquerda supriu a bolsa cloacal em 28 casos $(93,33 \%)$, destes a partir de um ramo em 21 casos $(70 \%)$ e dois ramos em sete casos $(23,33 \%)$. Já a artéria cloacal direita supriu a bolsa cloacal em 13 casos $(43,33 \%)$, com um ramo em 11 casos $(36,67 \%)$ e dois ramos em dois casos $(6,67 \%)$. A artéria cloacal

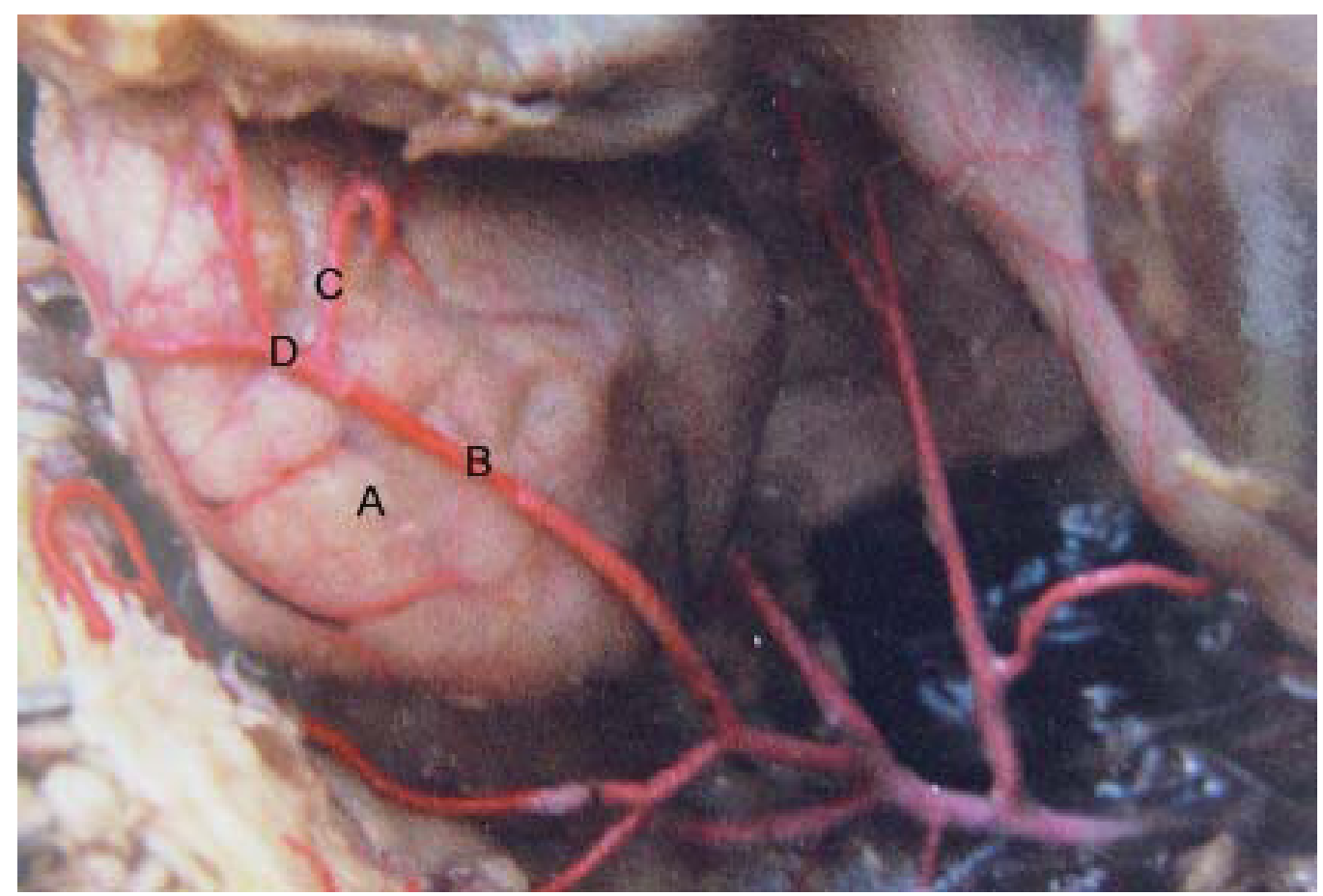

FIGURA 1: Cavidade celômica de ave (Gallus gallus) da linhagem Lhoman White LSL, demonstrando a bolsa cloacal (A), desviada para o antímero direito, e originando na artéria pudenda interna esquerda (B) as artérias bursocloacal esquerda (C) e cloacal esquerda (D). 
esquerda esteve presente em 15 casos (50\%) suprindo a bolsa cloacal, sendo que através de um ramo em 14 casos $(46,67 \%)$ e dois ramos em um caso $(3,33 \%)$.

Independentemente da origem, foram vistos de dois a seis ramos arteriais suprindo a bolsa cloacal. Assim, mais freqüentemente, estiveram presentes dois ramos em cinco casos (16,67\%), três ramos em 14 casos $(46,67 \%)$, quatro ramos em três casos $(10 \%)$, cinco ramos em seis casos $(20 \%)$ e seis ramos em um caso $(3,33 \%)$.

Em relação aos territórios de distribuição das artérias responsáveis pelo suprimento sangüíneo da bolsa cloacal, verificou-se que o quadrante caudal direito recebeu um ramo da artéria bursocloacal direita em 21 casos $(70 \%)$, um ramo da artéria cloacal direita em 10 casos $(33,33 \%)$, dois ramos da artéria cloacal direita em dois casos $(6,67 \%)$ e três ramos da artéria bursocloacal direita em dois casos $(6,67 \%)$.

O quadrante cranial direito foi atingido por um ramo da artéria bursocloacal direita em dois casos $(6,67 \%)$ e, ainda, por um ramo da artéria cloacal direita em um caso $(3,33 \%)$.

O quadrante caudal esquerdo recebeu um ramo da artéria bursocloacal esquerda em 21 casos $(70 \%)$, um ramo da artéria cloacal esquerda em 12 casos (40\%) e, ainda, dois ramos da artéria bursocloacal esquerda em seis casos $(20 \%)$.

Já o quadrante cranial esquerdo recebeu um ramo da artéria bursocloacal esquerda em um caso $(3,33 \%)$ e, ainda, um ramo da artéria cloacal esquerda em três casos $(10 \%)$.

Quando considerados conjuntamente, a origem, as associações e o número total de vasos que suprem cada um dos quadrantes, evidenciou-se uma disposição própria em cada um dos espécimes examinados.

No tocante às associações entre os vasos que suprem a bolsa cloacal, foi possível observar que, em 10 casos $(33,33 \%)$, as artérias bursocloacais direita e esquerda contribuem simultaneamente para o suprimento sangüíneo da bolsa cloacal, da mesma forma as artérias bursocloacais direita e esquerda, assim como ocorre com as artérias cloacais direita e esquerda, que forne- cem ramos para a bolsa cloacal em sete casos $(23,33 \%)$. Notou-se ainda a contribuição das artérias bursocloacais direita e esquerda e da artéria cloacal esquerda em cinco casos (16,67\%); já as artérias bursocloacais direita e esquerda e a artéria cloacal direita contribuíram para o suprimento sangüíneo da bolsa cloacal em outros cinco casos (16,67\%). Estiveram presentes ain$\mathrm{da}$, contribuindo para o suprimento da bolsa cloacal, as artérias bursocloacal direita e cloacal esquerda, em dois casos $(6,67 \%)$, e as artérias bursocloacal esquerda e as artérias cloacais direita e esquerda, em um caso $(3,33 \%)$.

\section{Discussão}

Os estudos relativos ao gênero Gallus, promovidos por Schwarze e Schröder (1970), Zamojska (1975), Nickel et al. (1981), Getty (1986) e Baumel (1993), retrataram de maneira superficial o comportamento vascular relativo ao suprimento sangüíneo para a bolsa cloacal. Neste mesmo contexto foram encontrados ainda os relatos de Baumel (1988), Scala et al. (1989) e Onyeanusi et al. (1993), respectivamente, para patos, pombos e galinha d'Angola. Todos esses informes mostraram-se muito genéricos, na medida que, no presente estudo, em aves da linhagem Lhoman White LSL, foram enfocados aspectos mais específicos, especialmente no que diz respeito à origem, número e território de distribuição das artérias que suprem a bolsa cloacal nessa linhagem de aves.

Por outro lado, verificou-se que informes pertinentes ao estudo sistemático relativo ao suprimento vascular sangüíneo da bolsa cloacal foram dados por Silva et al. (1997; 2001 e 2003), Santana et al. (1999; 2000 e 2003) e Lima et al. (2002), quando abordaram os arranjos daqueles vasos em diferentes linhagens de aves, tais como Cobb 500, Ross, NPK, Label Rouge, Petterson, Avian Farms e Hubbard.

No que tange às origens dos vasos que suprem a bolsa cloacal, no presente estudo foi possível verificar sistematicamente que este fato esteve na dependência das artérias bursocloacais e cloacais, em ambos os antímeros, coincidindo, portanto, com os dados fornecidos por Zamojska (1975), Nickel et al. (1981), Silva et al. 
(1997; 2001 e 2003), Santana et al. (1999; 2000 e 2003) e Lima et al. (2002). Sobretudo, tal como em Silva et al. (2001 e 2003) e Lima et al. (2002), foram evidenciados ramos das artérias cloacais, de ambos os antímeros, contribuindo para o suprimento da bolsa cloacal em aves da linhagem Lhoman White LSL.

A análise da literatura mostra que o suprimento arterial para a bolsa cloacal recebe contribuição também da artéria mesentérica caudal (Schwarze e Schröder, 1970; Silva et al., 1997 e 2003; Santana et al., 1999; 2000 e 2003; Lima et al., 2002), da artéria caudal mediana (Silva et al., 1997; Santana et al., 1999; 2000 e 2003; Lima et al., 2002) e da artéria ilíaca externa esquerda (Santana et al., 2003). No presente trabalho, a ausência de ramos destas artérias suprindo a bolsa cloacal pode ser explicado pelas particularidades anatômicas expressas na linhagem Lhoman White LSL, o que sugere que estas aves apresentam características específicas à linhagem. Da mesma forma, a ausência desses vasos não aparenta ter causado dano funcional ao referido órgão.

No concernente aos territórios ocupados pelas artérias que suprem a bolsa cloacal, observou-se que Scala et al. (1989), em patos (Anas platyrhyncus), comentaram sobre a presença de ramos das artérias pudendas internas penetrando pela margem lateral e face ventral do órgão. Já, de acordo com Silva et al. (1997; 2001 e 2003), Santana et al. (1999; 2000 e 2003) e Lima et al. (2002), em diferentes linhagens de Gallus gallus, a penetração dos ramos responsáveis pelo suprimento sangüíneo da bolsa cloacal ocorreu mais frequentemente na face ventral dos quadrantes caudais direito e esquerdo, seguido dos quadrantes craniais esquerdo e direito. Onyeanusi et al. (1993) comentaram que, na galinha d'Angola (Numida meleagris galeata), a penetração dos vasos ocorreu apenas sob a cápsula de revestimento do órgão, não mencionando as origens, tampouco o número de vasos arteriais destinados àquele órgão. Assim, diante dos achados do presente estudo, foi possível notar que o suprimento para a bolsa cloacal se deu mais freqüentemente pela disposição sintópica dos vasos responsáveis. Estabelecendo com isso parâmetros bastante precisos que justificam o estabelecimento de um padrão, juntamente com Silva et al. (1997, 2001 e
2003), Santana et al. (1999; 2000 e 2003) e Lima et al. (2002), para a disposição territorial dos ramos arteriais que suprem a bolsa cloacal no Gallus gallus.

Relativamente ao comportamento dos ramos arteriais responsáveis pelo suprimento da bolsa cloacal, verificou-se que estes atuam de maneira direta, ou seja, partem diretamente do tronco arterial para o órgão em questão, sendo os mesmos mencionados ainda, por Silva et al. (1997) e Santana et al. (2003), respectivamente em aves das linhagens Ross e Hubbard; aspecto esse também verificado neste estudo. Esses mesmos autores citaram ainda que a bolsa cloacal das linhagens Ross e Hubbard recebe ainda ramos indiretos dos seus vasos supridores. Assim, a presença de ramos diretos pode ser justificada pelo fato da bolsa cloacal ser o principal órgão do sistema linfático das aves, necessário assim um maior e mais efetivo aporte sangüíneo arterial.

Em relação ao número de ramos arteriais que suprem a bolsa cloacal, independentemente de suas origens, os achados do presente estudo foram de encontro às alusões de Silva et al. (1997; 2001 e 2003) e Santana et al. (1999 e 2000), onde esses ramos variaram em número de dois a seis, ao passo que a freqüência variou entre os ramos presentes de maneira bastante diversificada. Por outro lado, verificou-se nos achados de Santana et al. (2003) que esse número variou de dois a nove ramos, em aves da linhagem Hubbard, o que caracterizou esses animais de maneira diferenciada, principalmente pelo fato de que, naquelas, a freqüência desses ramos mostrou-se quase que constante em relação aos ramos cedidos pela artéria bursocloacal.

\section{Referências}

Baumel, J. J. 1988. Functional morphology of the apparatus of the pigeon (Columba livia). Advanced Anatomy, Embriology and Cell Biology, 110: 115-117.

Baumel, J. J. 1993. Handbook of avian anatomy: Nomina Anatomica Avium. $2^{\text {nd }}$ ed. Cambridge, Nuttall Ornithological Club, USA, 779pp.

Getty, R. 1986. Sisson/Grossman anatomia dos animais domésticos. $5^{\text {a }}$ ed. Guanabara Koogan, Rio de Janeiro, Brasil, 2000pp.

Lima, E. M. M.; Silva, F. O. C.; Severino, R. S.; Drummond, S. S.; Campos, D. B.; Bombonato, P. P.; Santana, M. I. S. 2002. Irrigação da bolsa cloacal em aves (Gallus gallus) da linhagem Cobb 500. Anais do XX Congresso Brasileiro de Anatomia, Maceió, Brasil, p.81. 
Nickel, R.; Schummer, A.; Seiferle, E. 1981. Anatomy of the domestic birds. Verlag Paul Parey, Berlin, Germany, 202pp.

Onyeanusi, B.; Ezeokoli, C. D.; Onyeanusi, J. C.; Ema, A. N. 1993. The anatomy of cloacal bursa (bursa of Fabricius) in the helmet guinea fowl (Numidea meleagris galeata). Anatomia Histologia Embryologia, 22: 212-221.

Payne, L. N. 1971. The lymphoid system. In: Bell, D. J. \& Freman, B. D. (eds). Physiology and biochemistry of the domestic fowl. v.2. Academic Press, London, UK, p. 985-1037.

Rosskpof, W.; Woerpel, R. 1966. Diseases of cage and aviary birds. $3^{\text {rd }}$ ed. Williams \& Wilkins: New York, USA, 365pp.

Santana, M. I. S.; Silva, F. O. C.; Severino, R. S.; Bombonato, P. P.; Marçal, A. V. 2003. Irrigação da bolsa cloacal em Gallus gallus domesticus (matrizes de corte Hubbard). Brazilian Journal of Veterinary Research and Animal Science, 40: 199-204.

Santana, M. I. S.; Silva, F. O. C.; Severino, R. S.; Santos, A. L. Q.; Drummond, S. S.; Bombonato, P. P. 1999. Irrigação da bolsa cloacal em aves reprodutoras da linhagem Peterson (Gallus gallus domesticus). Brazilian Journal of Veterinary Research and Animal Science, 36: sem paginação.

Santana, M. I. S.; Silva, F. O. C.; Severino, R. S.; Santos, A. L. Q.; Drummond, S. S.; Bombonato, P. P. 2000. Vascularização arterial da bolsa cloacal em Gallus gallus domesticus (matrizes de corte Avian Farms). Brazilian Journal of Veterinary Research and Animal Science, 37: 115-120.
Scala, G.; Caputo, G.; Paino, G.; Pelalalli, G. V. 1989. The vascularization of the bursa cloacalis (of Fabricius) in the duck. Anatomia Histologia Embryologia, 18: 66-75.

Schwarze, E.; Schröder, L. 1970. Compendio de anatomia veterinaria. v.5. Acribia, Milano, Itália, 372pp.

Silva, F. O. C.; Severino, R. S.; Drummond, S. S.; Bombonato, P. P.; Santana, M. I. S.; Lima, E. M. M. 2001. Irrigação da bolsa cloacal em aves (Gallus gallus Linnaeus, 1758) da linhagem NPK. Arquivos de Ciências da Saúde da Unipar, 5: 17-24.

Silva, F. O. C.; Severino, R. S.; Drummond, S. S.; Machado, G. V.; Bombonato, P. P.; Santana, M. I. S.; Lima, E. M. M. 2003. Suprimento arterial para a bolsa cloacal de galinhas (Gallus gallus Linnaeus, 1758) da linhagem Label Rouge. Archives of Veterinary Science, 8: 13-18.

Silva, F. O. C.; Severino, R. S.; Santos, A. L. Q.; Drummond, S. S.; Bombonato, P. P.; Santana, M. I. S.; Gonçalez, P. O.; Marçal, A. V. 1997. Vascularização arterial da bolsa cloacal em Gallus gallus domesticus (Linhagem Ross). Revista da Faculdade de Zootecnia, Veterinária e Agronomia de Uruguaiana, 4: 67-74.

Zamojska, D. 1975. Anatomical studies on the vascularization of the bursa of Fabricius and Uropigeal gland in the hens (Gallus domesticus). Part II. Blood vessels of the bursa of Fabricius (Bursa of Fabricii). Zoologica Poloniae, 24: 455-476. 